ARTICLE

Received 17 Mar 2014 | Accepted 14 Jul 2014 | Published 14 Aug $2014 \quad$ DOl: 10.1038/ncomms5685

\title{
Growing dynamical facilitation on approaching the random pinning colloidal glass transition
}

Shreyas Gokhale ${ }^{1, \star}$, K. Hima Nagamanasa ${ }^{2, \star}$, Rajesh Ganapathy ${ }^{3}$ \& A.K. Sood ${ }^{1,3}$

Despite decades of research, it remains to be established whether the transformation of a liquid into a glass is fundamentally thermodynamic or dynamic in origin. Although observations of growing length scales are consistent with thermodynamic perspectives, the purely dynamic approach of the Dynamical Facilitation (DF) theory lacks experimental support. Further, for vitrification induced by randomly freezing a subset of particles in the liquid phase, simulations support the existence of an underlying thermodynamic phase transition, whereas the DF theory remains unexplored. Here, using video microscopy and holographic optical tweezers, we show that DF in a colloidal glass-forming liquid grows with density as well as the fraction of pinned particles. In addition, we observe that heterogeneous dynamics in the form of string-like cooperative motion emerges naturally within the framework of facilitation. Our findings suggest that a deeper understanding of the glass transition necessitates an amalgamation of existing theoretical approaches.

\footnotetext{
${ }^{1}$ Department of Physics, Indian Institute of Science, Bangalore 560012, India. ${ }^{2}$ Chemistry and Physics of Materials Unit, Jawaharlal Nehru Centre for Advanced Scientific Research, Jakkur, Bangalore 560064, India. ${ }^{3}$ International Centre for Materials Science, Jawaharlal Nehru Centre for Advanced Scientific Research, Jakkur, Bangalore 560064, India. * These authors contributed equally to this work. Correspondence and requests for materials should be addressed to S.G. (email: gokhale@physics.iisc.ernet.in) or to K.H.N. (email: hima@jncasr.ac.in).
} 
$\mathrm{n}$ spite of significant theoretical ${ }^{1,2}$ and experimental ${ }^{3,4}$ advances since the pioneering work of Adam and Gibbs ${ }^{5}$, it is as yet unclear whether the glass transition is thermodynamic or dynamic in nature. This is predominantly because supercooled liquids fall out of equilibrium well before the putative phase transition, be it dynamic or thermodynamic, is reached. This limitation has spurred the search for alternate ways of approaching the glass transition ${ }^{6-8}$, of which random pinning has attracted widespread interest in the last 2 years. Random pinning has mainly been studied within the framework of the Random First-Order Transition (RFOT) theory ${ }^{9,10}$, which predicts a thermodynamic 'ideal' glass transition at a finite fraction of pinned particles, for temperatures above the Kauzmann temperature of the unconstrained liquid ${ }^{7}$. By comparison, the contrasting perspective offered by the Dynamical Facilitation (DF) theory, 11 has received far less attention. Recently, it was shown that kinetically constrained spin models ${ }^{12}$, which form the conceptual basis of the DF theory, do not exhibit a thermodynamic phase transition with random pinning ${ }^{13}$. Nonetheless, a systematic analysis of facilitation in real glass-formers with random pinning is yet to be undertaken, and is imperative in order to gain a deeper understanding of the glass transition.

The DF theory postulates that structural relaxation is dictated by the concerted motion of localized mobile defects, termed excitations, and the slowdown of dynamics is attributed to a reduction in the concentration of these excitations. Here, we investigate these postulates experimentally, by performing optical video microscopy on dense binary hard-sphere like colloidal suspensions. From the analysis of particle trajectories, we identify and characterize excitations and find that they are localized in space and time. Further, we observe that the concentration of these excitations indeed decreases on approaching the glass transition. Moreover, by computing facilitation volumes and mobility transfer functions, we demonstrate that DF grows on approaching the glass transition. We also show that string-like cooperative motion emerges from the correlated dynamics of excitations. Most importantly, by using holographic optical tweezers to pin a subset of particles, we show that DF plays a significant role in governing structural relaxation, even when the glass transition is approached by increasing the concentration of pinned particles.

\section{Results}

Excitations are localized in space and time. We first investigated DF for the unpinned case, in a binary colloidal glass-forming liquid composed of silica particles (see the section 'Experimental Details' in Methods). To identify excitations, we employed the protocol developed in ref. 14. A particle was said to be associated with an excitation of size $a$ and 'instanton' time $\Delta t$, if it underwent a displacement of magnitude $a$ over a time interval $\Delta t$, and persisted in its initial and final positions for at least $\Delta t$. In practice, excitations were identified by defining for the coarsegrained trajectory $\overline{\mathbf{r}}_{i}(t)$ of every particle $i$, the functional

$$
h_{i}\left(t, t_{a} ; a\right)=\prod_{t^{\prime}=t_{a} / 2-\Delta t}^{t_{a} / 2} \theta\left(\left|\overline{\mathbf{r}}_{i}\left(t+t^{\prime}\right)-\overline{\mathbf{r}}_{i}\left(t-t^{\prime}\right)\right|-a\right)
$$

Here, $\theta(x)$ is the Heaviside step function and $t_{a}$, known as the commitment time, is typically chosen to be approximately three or four times the mean value of $\Delta t$ for an excitation of size $a$ (see the section 'Identifying Excitations' in Methods. Also see inset to Fig. 1a). To obtain sufficient statistics, we restricted our analysis to $a=0.5 \sigma_{\mathrm{S}}$. We observe that the distributions of instanton times $P_{a}(\Delta t)$ for different area fractions $\phi$ nearly collapse onto the same curve and the mean instanton time $\langle\Delta t\rangle$ remains almost constant (Fig. 1a). Also, at large $\phi,\langle\Delta t\rangle$ is much smaller than the structural relaxation time $\tau_{\alpha}$ (Supplementary Fig. 1), which clearly shows that excitations are localized in time, in accordance with ref. 14.

To measure the spatial extent of excitations, we first computed the function

$$
\begin{aligned}
\mu\left(r, t, t^{\prime} ; a\right)= & \frac{1}{\left\langle h_{1}\left(0, t_{a} ; a\right)\right\rangle}\left\langle h_{1}\left(0, t_{a} ; a\right) \sum_{i \neq 1}^{N}\left|\overline{\mathbf{r}}_{i}\left(t^{\prime}\right)-\overline{\mathbf{r}}_{i}(t)\right|\right. \\
& \left.\delta\left(\overline{\mathbf{r}}_{i}(t)-\overline{\mathbf{r}}_{1}(t)-\overline{\mathbf{r}}\right)\right\rangle
\end{aligned}
$$

For $t=-t_{a} / 2$ and $t^{\prime}=t_{a} / 2, \mu\left(r, t, t^{\prime} ; a\right)$ yields the displacement density at a distance $r$ from an excitation of size $a$ located at the origin at time $t=0$, over a time interval $t_{a}$ centred on 0 . From $\mu\left(r, t, t^{\prime} ; a\right)$, the spatial extent of excitations was then extracted by defining the function

$$
F(r ; a)=\frac{\mu\left(r,-t_{a} / 2, t_{a} / 2 ; a\right)}{g(r) \mu_{\infty}\left(t_{a}\right)}-1
$$

where $g(r)$ is the radial pair-correlation function and $\mu_{\infty}\left(t_{a}\right)=$ $\left\langle\left|\overline{\mathbf{r}}_{i}\left(t+t_{a}\right)-\overline{\mathbf{r}}_{i}(t)\right|\right\rangle . F(r ; a)$ decays within 8 particle diameters irrespective of $\phi$ (Fig. 1b), confirming that excitations are spatially localized objects that do not grow on approaching the glass transition ${ }^{14}$.

Evidence for growing DF. A visual indicator of the concentration of excitations $c_{a}$ is the particles' displacement field computed over $\langle\Delta t\rangle=12 \mathrm{~s}$ (Fig. 1c-e). The decreasing fraction of particles with displacements greater than $a$ (maroon spheres) observed in Fig. 1c-e strongly indicates a lowering in $c_{a}$ with increasing $\phi$. More quantitatively, the concentration of excitations, formally defined as

$$
c_{a}=\left\langle\frac{1}{V t_{a}} \sum_{i=1}^{N} h_{i}\left(0, t_{a} ; a\right)\right\rangle
$$

where $V$ is the volume and $N$ is the total number of particles, indeed decreases with $\phi$, in accordance with the DF theory (Fig. 1f).

The second major ingredient of the DF theory is facilitation, which assumes that new excitations primarily occur in the vicinity of existing excitations. To quantify the degree of facilitation in our system, we computed the facilitation volume

$$
v_{\mathrm{F}}(t)=\int\left[\frac{\mu\left(r, t_{a} / 2, t ; a\right)}{g(r) \mu_{\infty}\left(t-t_{a} / 2\right)}-1\right] d \mathbf{r}
$$

We observe that for all $\phi$ s considered, $v_{\mathrm{F}}(t)$ initially increases with time, reaches a maximum value $v_{\mathrm{F}}^{\max }$ at time $t_{\max }$ and then decreases at longer times (Fig. 1g). Moreover, both $t_{\max }$ and $v_{\mathrm{F}}^{\max }$ increase with $\phi$ (Fig. 1g), in complete agreement with recent simulations of atomistic glass-formers ${ }^{14}$ as well as spin models ${ }^{15}$.

Although the presence of facilitated dynamics has been observed in numerical simulations ${ }^{14,16-18}$ and experiments on driven granular media 19,20 , its relative importance as a mechanism of structural relaxation is still debated. Some studies claim that facilitation becomes increasingly important on approaching the glass transition ${ }^{14,17,18}$, whereas others maintain that its relevance diminishes ${ }^{20}$. Within RFOT, facilitation is a by-product of slow dynamics rather than its chief cause $\mathrm{e}^{21}$. To examine the importance of DF in our system, we computed the mobility transfer function $M(\Delta \tau)$ as defined in ref. 17 (see the section 'Mobility transfer function' in Methods for a definition of $M(\Delta \tau)$. We observe that analogous to ref. 17, $M(\Delta \tau)$ for various $\phi$, computed separately for large and small silica particles, 

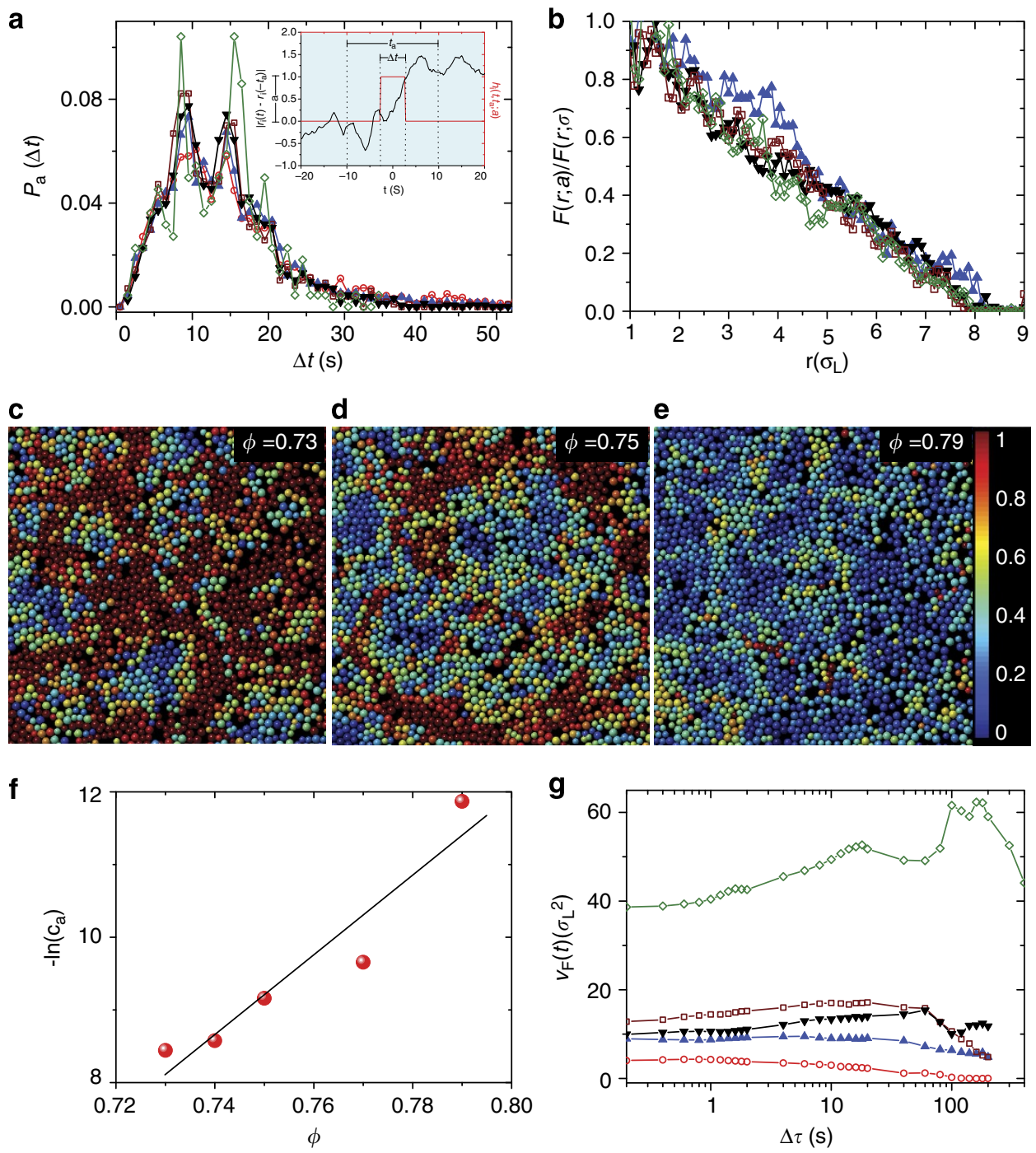

Figure 1 | Dynamical facilitation in the absence of pinning. a) Distribution of instanton times $P_{a}(\Delta t)$ for $\phi=0.73$ (open red circles), $\phi=0.74$ (filled blue triangles), $\phi=0.75$ (filled black inverted triangles), $\phi=0.77$ (open brown squares) and $\phi=0.79$ (open green diamonds), showing that excitations are localized in time. (Inset to a) Representative sub-trajectory of a particle coarse-grained over $2 \mathrm{~s}$, shown in black, and the corresponding functional $h_{i}\left(t, t_{a} ; a\right)$ shown in red. The instanton time duration $\Delta t$ and the commitment time $t_{a}$ are marked by dotted lines. The displacement is measured in units of $a=0.5 \sigma_{\mathrm{S}}$ and the trajectory has been shifted arbitrarily along the $y$ axis to make the rise in displacement coincide with region of non-zero $h_{i}\left(t, t_{a} ; a\right)$. (b) The function $F(r ; a)$ normalized by its value at $r=\sigma_{\mathrm{L}}$ for $\phi=0.74$ (filled blue triangles), $\phi=0.75$ (filled black inverted triangles), $\phi=0.77$ (open brown squares) and $\phi=0.79$ (open green diamonds), showing that excitations are localized in space. (c-e) Displacement field of particles for $\phi=0.73$ (c), $\phi=0.75$, (d) and $\phi=0.79$ (e). The colourbar corresponds to particle displacements normalized by the excitation size $a$. Particles coloured in maroon indicate displacements $\geq a$. (f) Concentration of excitations $c_{a}$ vs $\phi$. (g) Facilitation volume $v_{\mathrm{F}}(t)$ for various $\phi$ s. The symbols and colours in $\mathbf{g}$ are same as those in $\mathbf{a}, \mathbf{b}$.

a

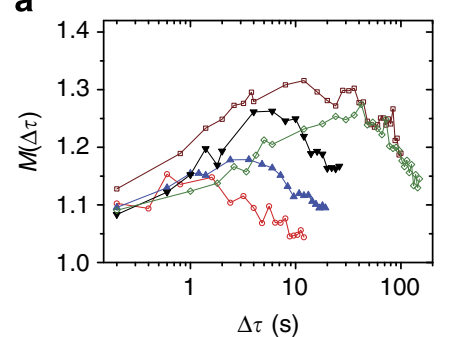

b

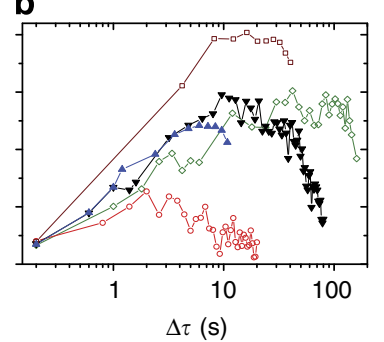

c

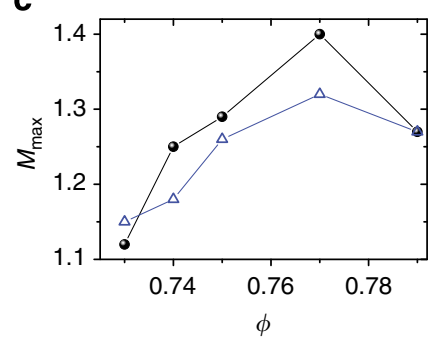

Figure 2 | Mobility transfer function (a,b). The mobility transfer function $M(\Delta \tau)$ for small (a) and large (b) silica particles for $\phi=0.73$ (open red circles), $\phi=0.74$ (filled blue triangles), $\phi=0.75$ (filled black inverted triangles), $\phi=0.77$ (open brown squares) and $\phi=0.79$ (open green diamonds). (c) Maximum of $M(\Delta \tau), M_{\max }$ as a function of $\phi$ for small (open blue triangles) and large (filled black spheres) particles. 
exhibits a maximum at a time comparable to the cage-breaking time (Fig. 2a,b). $M_{\max }$ is expected to increase over the regime in which facilitation is dominant, and decrease in the regime where other delocalized activated processes dictate relaxation ${ }^{15}$. We find that for $0.73 \leq \phi<0.79, M_{\max }$ increases systematically, showing that DF plays an important role over this range of $\phi$ (Fig. 2c). Interestingly, at $\phi=0.79, M_{\max }$ shows a decrease relative to its value at $\phi=0.77$ (Fig. 2c). We note that the typical distance travelled by most mobile particles over the cage-breaking time is $\sim 0.5 \sigma \mathrm{s}$, which corresponds to the size $a$ of the excitations considered in this work. For $\phi=0.79$, the mean separation between these excitations, $l_{a}=\left(t_{a} c_{a}\right)^{-1 / 2} \quad(\sim 40 \mu \mathrm{m})$, is comparable to the system size $(\sim 49 \mu \mathrm{m})$, whereas for all other $\phi \mathrm{s}$, it is much smaller $(<13 \mu \mathrm{m})$. The observed decrease in $M_{\max }$ at $\phi=0.79$ may therefore be a consequence of finite size effects ${ }^{15}$, although similar behaviour can stem from other delocalized activated relaxation processes. Nonetheless, the fact that $M_{\max }$ remains significantly larger than 1 , together with the substantial growth in facilitation volume shows that DF significantly contributes to structural relaxation over the range of $\phi$ considered in this work (see Supplementary Movie 1 for a visualization of facilitated dynamics).

Correspondence between excitations and microstrings. Consistent with RFOT and the Adam-Gibbs theory, a considerable body of evidence supporting the existence of growing dynamic length scales on approaching the glass transition ${ }^{22-26}$ has accumulated over the last two decades. It is natural to wonder if these growing dynamic correlations are influenced by DF. To investigate this possibility, we explored the connection between localized excitations and string-like cooperative motion of mobile particles $24,27,28$, which is believed to be consistent with RFOT $^{29,30}$. We constructed strings from the top $10 \%$ most mobile particles using the procedure described in ref. 24. Figure 3a shows a representative string composed of 13 particles for $\phi=0.79$, for which $\Delta \tau=t^{*}=66 \mathrm{~s}$, where $t^{*}$ corresponds to the maximum of the non-Gaussian parameter $\alpha_{2}(t)$ (ref. 22). From their trajectories over $t^{\star}$, the particles constituting the string can be divided into three independent groups, termed 'microstrings' 31 , such that motion within each microstring is coherent (Fig. $3 \mathrm{~b}-\mathrm{d}$ ). We observe that in one of the microstrings (Fig. 3d), each particle $i$ is associated with an excitation for some time $\Delta t_{i}<t^{\star}$ (Fig. 3e), which strongly suggests that over $t^{\star}$, the motion in the other microstrings is facilitated by these excitations. Moreover, the maximum string length $n_{\mathrm{s}}$ increases with the mean separation between excitations (Supplementary Fig. 2). Collectively, these findings show that string-like cooperative motion at longer times emerges in a hierarchical manner, from the short-time dynamics of excitations ${ }^{14}$.

Evolution of facilitation with random pinning. In the presence of random pinning, numerical evidence based on static quantities supports the RFOT scenario ${ }^{32}$, whereas that based on dynamic ones does not ${ }^{33}$. However, the rapid growth of relaxation times with increasing fraction of pinned particles is undisputed ${ }^{7,32,33}$. It is thus natural to ask whether DF accounts for the observed slowdown of dynamics. Indeed, as the DF theory is a parallel approach to RFOT, a systematic investigation of facilitation in the context of random pinning is highly desirable ${ }^{7}$ and is likely to enrich our understanding of the glass transition. We therefore explored the impact of DF on structural relaxation in the presence of random pinning. Towards this end, we imaged a binary mixture of polystyrene particles at fixed $\phi$, and used holographic optical tweezers to pin a subset of particles in the field of view (Fig. 4a. Also see the sections 'Experimental details' and 'Estimation of the trap potential' in Methods. See Supplementary Movie 2 for a visualization of glasst dynamics in the presence of pinned particles). The fraction of pinned particles $f_{\mathrm{p}}$ was varied from 0.03 to 0.26 . We see that even at relatively low values of $\phi$ and $f_{\mathrm{p}}$, the pinned particles have a significant impact on the dynamics of the remaining free particles. The structural relaxation time $\tau_{\alpha}$ increases by a factor of 4 relative to its value in the unpinned case (Fig. $4 \mathrm{~b}$ ) for $\phi=0.71$ when $f_{\mathrm{p}}$ is varied from 0.03 to 0.12 . Further experiments at higher values of $\phi$ and $f_{\mathrm{p}}$ reveal that in concord with recent simulations ${ }^{32,33}$, the slowdown in dynamics with pinning becomes increasingly pronounced at larger $\phi$ s (Fig. 4b). Most importantly, at $\phi \geq 0.74$ and $f_{\mathrm{p}} \geq 0.21$, the system becomes non-ergodic over experimental time scales, as
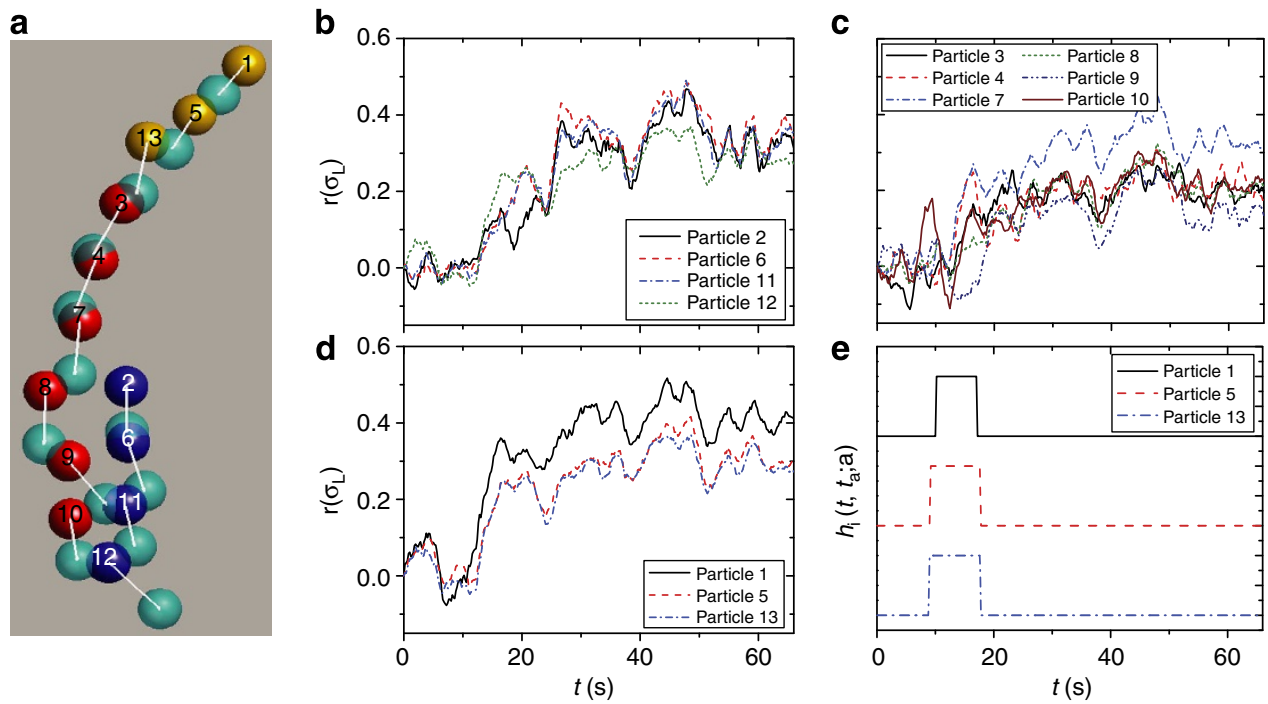

Figure 3 | Connection between excitation dynamics and microstrings. (a) Schematic of a representative string composed of 13 particles for $\phi=0.79$ constructed over a time interval $\Delta \tau=t^{*}=66 \mathrm{~s}$. Particles in yellow, red and blue correspond to initial positions of particles belonging to three independent microstrings. Cyan spheres represent final positions of the particles. (b-d) Trajectories of particles corresponding to the blue (b), red (c) and yellow (d) microstrings over time $t^{\star}$. In $\mathbf{b}$-d, the particle labels are identical to those in $\mathbf{a}$. (e) The functional $h_{i}\left(t, t_{a} ; a\right)$, where $a=0.3 \sigma_{\mathrm{s}}$, corresponding to the trajectories shown in $\mathbf{d}$ for the yellow microstring in $\mathbf{a}$. 
a

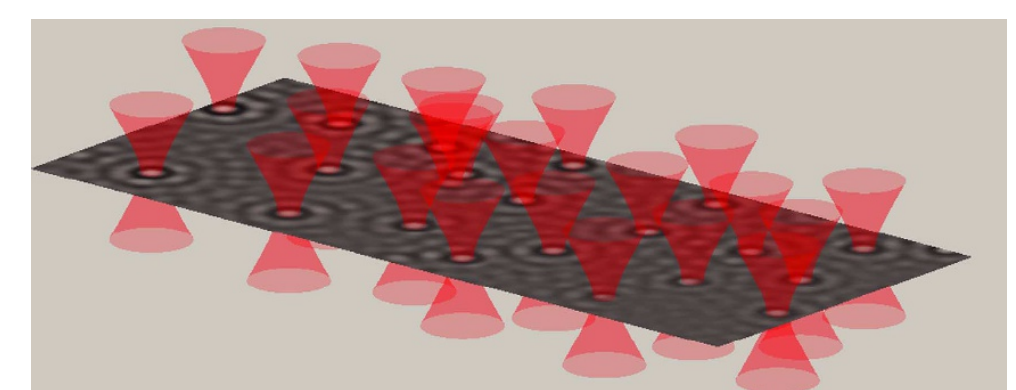

b

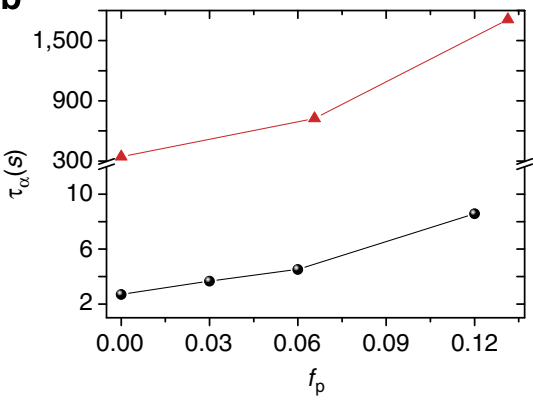

d

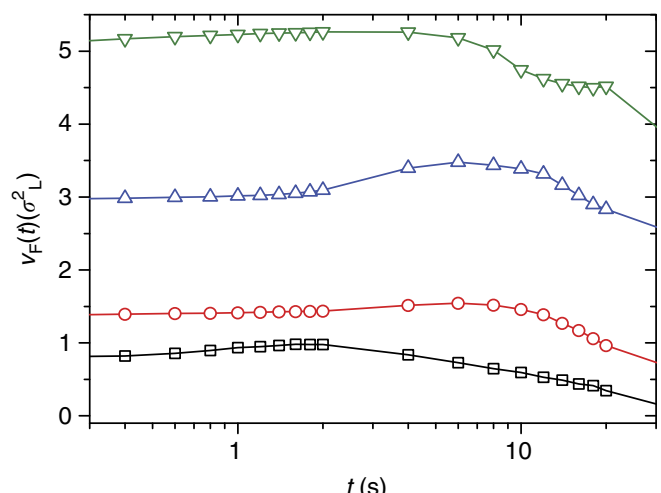

C
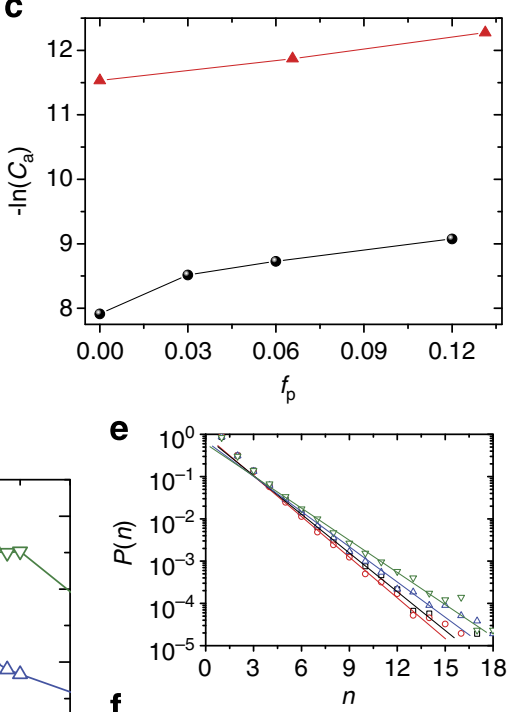

f

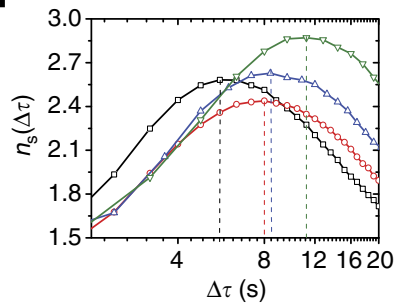

Figure 4 | Effect of random pinning. (a) Schematic of the trapping potentials (shown in red) created by the holographic optical tweezers. The underlying image represents a small portion of the field of view for $f_{p}=0.06$ and $\phi=0.71$. The image has been generated by averaging over $\sim 15 \tau_{\alpha}$. Pinned particles appear bright because of their low mobility and high overlap with initial positions. (b) Structural relaxation time $\tau_{\alpha}$ for free particles as a function of $f_{\mathrm{p}}$ for $\phi=0.71$ (filled black spheres) and $\phi=0.74$ (filled red triangles). Note the break in the $\tau_{\alpha}$ axis. (c) Concentration of excitations $c_{a}$ as a function of $f_{\mathrm{p}}$ for $\phi=0.71$ (filled black spheres) and $\phi=0.74$ (filled red triangles). In $\mathbf{b}$ and $\mathbf{c}$, only those data points for which $F_{\mathrm{s}}\left(q_{0}, t\right)$ decays at least to $1 /$ e are shown. (d) Facilitation volume $v_{\mathrm{F}}(t)$ for $f_{\mathrm{p}}=0$ (open black squares), $f_{\mathrm{p}}=0.03$ (open red circles), $f_{\mathrm{p}}=0.06$ (open blue triangles) and $f_{\mathrm{p}}=0.12$ (open green inverted triangles). (e) Distribution of string lengths $P(n)$ for $\Delta \tau$ corresponding to the maximum string length and (f) string length $n_{\mathrm{s}}(\Delta \tau)$ for various $f_{\mathrm{p}}$. In $\mathbf{d}-\mathbf{f}, \phi=0.71$. The colours and symbols in $\mathbf{e}$ and $\mathbf{f}$ are identical to those in $\mathbf{d}$.

evident from the profile of the self-intermediate scattering function $F_{s}\left(q_{0}, t\right)$ (Supplementary Fig. 3. Also see Supplementary Movie 3 for a visualization of the slowdown of dynamics with $f_{\mathrm{p}}$ ). These results indicate the possible existence of a glass transition induced by random pinning. To verify whether the observed increase in $\tau_{\alpha}$ with $f_{\mathrm{p}}$ is a consequence of growing facilitation, we repeated the analysis shown in Fig. 1 for the pinned case. At $\phi=0.71$, we observe that the concentration of excitations $c_{a}$ decreases by more than a factor of 3 with $f_{\mathrm{p}}$ (Fig. 4c), whereas the facilitation volume $v_{\mathrm{F}}(t)$ shows a concomitant increase (Fig. $4 \mathrm{~d}$ ), for $f_{\mathrm{p}}$ ranging from 0.03 to 0.12 . We observe a similar evolution of $c_{a}$ (Fig. 4c) and $v_{\mathrm{F}}(t)$ (Supplementary Fig. 4) with $f_{\mathrm{p}}$ for higher $\phi$ s as well. These results show that in striking resemblance to the unpinned case (Fig. 1f,g), DF plays a significant role even in the context of the random pinning glass transition.
Next, we examined the interplay between facilitation and string-like cooperative motion in the presence of random pinning. We observe that the behaviour of string-like cooperative motion as a function of $f_{\mathrm{p}}$ is remarkably similar to its variation with $\phi$. In particular, the distribution of string lengths $P(n)$ is nearly exponential (Fig. 4e), and the average string length $n_{\mathrm{s}}=$ $\Sigma n^{2} P(n) / \Sigma n P(n)$ exhibits a maximum as a function of time (Fig. 4f. See Supplementary Fig. 2 for corresponding results in the unpinned case). Further, the time at which $n_{\mathrm{s}}$ reaches a maximum, $\Delta \tau_{\max }$, increase systematically with $f_{\mathrm{p}}$ (Fig. 4f). However, in contrast to the unpinned case, the maximal string length $n_{\mathrm{s}}^{\max }$ does not grow systematically with increasing $f_{\mathrm{p}}$ (Fig. $4 \mathrm{f}$ and Supplementary Fig. 4). Interestingly, the four-point dynamic susceptibility $\chi_{4}\left(a_{\mathrm{p}}, t\right)$ (see the section 'Dynamic susceptibility' in Methods for a definition) does not exhibit a systematic increase with $f_{\mathrm{p}}$ (Supplementary Fig. 5), which is consistent with ref. 33 as 
well as the dependence of string lengths on $f_{\mathrm{p}}$ (Fig. $4 \mathrm{f}$ ). Although DF in known to result in the growth of $\chi_{4}\left(a_{\mathrm{p}}, t\right)$ on approaching the glass transition in the absence of pinning 1 , a correspondence between facilitation and $\chi_{4}\left(a_{\mathrm{p}}, t\right)$ in the context randomly pinned particulate systems is yet to be established. Our findings therefore warrant investigation in future experiments and simulations.

\section{Discussion}

Our experiments have highlighted the importance of DF on approaching the colloidal glass transition, be it driven by density or random pinning. Although the procedure to characterize excitations employed here involves the analysis of particle displacements, it has been speculated ${ }^{15}$ that localized soft modes $^{16}$ may provide a method of identifying excitations, without recourse to their dynamics. This is a very interesting possibility that needs to be investigated experimentally as well as numerically. Yet another promising avenue is the development of amorphous order on approaching the glass transition. Although the DF theory makes no predictions for structural evolution on approaching the glass transition, signatures of growing amorphous order in the form of overlap functions $s^{32,34}$ and point-to-set correlations $s^{35,36}$ have been observed in numerical simulations. It is of paramount importance to examine whether DF can be reconciled with the growth of these static correlations. In particular, it would be worthwhile to probe whether facilitation plays a role in the generic decoupling of static and dynamic length scales ${ }^{26,37}$. Finally, we expect our observations to stimulate theoretical and numerical research on dynamic space-time phase transitions between mobile liquid and immobile glassy states ${ }^{6,38}$ in the presence of random pinning. Specifically, as $c_{a}$ is known to be an order parameter for such space-time phase transitions ${ }^{39}$, the observed decrease in $c_{a}$ with $f_{\mathrm{p}}$ (Fig. $4 \mathrm{c}$ ) implies that changing $f_{\mathrm{p}}$ influences the system's proximity to a dynamic phase transition. It would be fascinating to examine whether or not this dynamic phase transition coincides with the thermodynamic one predicted by $\mathrm{RFOT}^{7}$. We expect a confluence of experimental, theoretical and numerical research aimed at exploring these questions to emerge in the wake of our findings.

\section{Methods}

Experimental details. For experiments without pinning, the system comprised of $N_{\mathrm{L}}$ large and $N_{\mathrm{S}}$ small silica colloids of diameters $\sigma_{\mathrm{L}}=970 \mathrm{~nm}$ and $\sigma_{\mathrm{S}}=760 \mathrm{~nm}$, respectively. The size ratio $\sigma_{\mathrm{L}} / \sigma_{\mathrm{S}}=1.3$ and the number ratio $N_{\mathrm{L}} / N_{\mathrm{S}}=0.66$ effectively suppressed crystallization. Dilute samples were loaded in a wedge shaped cell ${ }^{40}$ and the area fraction $\phi$ was tuned systematically by controlled sedimentation of particles to the monolayer thick region of the wedge. For experiments with pinning, we used a binary mixture of polystyrene spheres of diameters 1.4 and $1.05 \mu \mathrm{m}$. For $\phi=0.71$, the number ratio $N_{\mathrm{L}} / N_{\mathrm{S}}$ was 2.3 , whereas for experiments at larger $\phi$, this ratio was reduced to 0.7 in order to enhance the frustration to crystallization. Both silica and polystyrene particles interact via shortranged repulsive screened Coulomb interactions with a Debye length that is typically smaller than $10 \%$ of the particle diameter. To a good approximation, therefore, these systems can be treated as suspensions of effective hard spheres. The holographic optical tweezers set up consisted of a linearly polarized constant power $(800 \mathrm{~mW}) \mathrm{CW}$ laser (Spectra-Physics, $\lambda=1,064 \mathrm{~nm})$. A spatial light modulator (Boulder Nonlinear Systems, Inc.) was used to generate upto 200 traps at randomly chosen locations. Only large polystyrene particles were pinned. The laser was switched on after fixing the trap positions and equilibrating the sample to ensure simultaneous activation of the traps. The average power per trap $(\sim 0.5 \mathrm{~mW})$ was sufficient to ensure that the pinned particles did not escape over the duration of the experiment, as evidenced by the difference in mean squared displacements of free and pinned particles (Supplementary Fig. 6). For experiments with and without pinning, samples were imaged using a Leica DMI 6000B optical microscope with a $\times 100$ objective (Plan apochromat, numerical aperture $=1.4$, oil immersion) and images were captured at frame rates ranging from 2.5 to 5 f.p.s. for $1-3 \mathrm{~h}$ depending on the values of $\phi$ and $f_{\mathrm{p}}$. The $66 \times 49 \mu \mathrm{m}^{2}$ field of view typically contained $>4,000$ particles for the silica sample and $\sim 1,200$ particles for the polystyrene sample. For experiments in which a maximum $f_{\mathrm{p}} \geq 0.21$ was reached, a restricted field of view of $34 \times 37 \mu \mathrm{m}^{2}$ containing $\sim 600$ polystyrene particles was used. Standard algorithms were used for particle tracking ${ }^{41}$ and quantities of interest were extracted using codes developed in-house.
Identifying excitations. As the functional $h_{i}\left(t, t_{a} ; a\right)$ does not impose an upper bound on the size of excitations, the algorithm detects excitations of size greater than $a$, if their $\Delta t$ is sufficiently small. These anomalously large excitations were ignored, in order to ensure that they do not bias the analysis. To calculate $h_{i}\left(t, t_{a} ; a\right)$, the initial guess for $\Delta t, \Delta t_{\text {in }}$, was chosen by manually inspecting a few trajectories, and the corresponding initial value of $t_{a}$ was set to be slightly larger than $3 \Delta t_{\text {in. }}$. From these initial guesses, we computed $h_{i}\left(t, t_{a} ; a\right)$ and plotted a distribution of $\Delta t$. The mode $\Delta t_{\mathrm{m}}$ of this distribution was chosen as the initial value of $\Delta t$ for the final computation of $h_{i}\left(t, t_{a} ; a\right)$, and $t_{a}$ was accordingly set to a value slightly greater than $3 \Delta t_{\mathrm{m}}$. The results are insensitive to the choice of $\Delta t$, but depend strongly on $t_{a}$.

Mobility transfer function. Given two successive time intervals $\Delta t_{1}$ and $\Delta t_{2}$ of a fixed duration such that $\Delta t_{1}=\Delta t_{2}=\Delta \tau$, the mobility transfer function $M(\Delta \tau)$ quantifies the excess probability for a mobile particle in $\Delta t_{2}$ to be located close to a mobile particle in $\Delta t_{1}$, relative to the probability that it is located near an immobile particle. To compute $M(\Delta \tau)$, we identified the top $10 \%$ most mobile particles for two successive intervals of duration $\Delta \tau$ and generated the distribution $P_{\mathrm{m}}(r, \Delta \tau)$ of the minimum distance of each mobile particle in the second interval from the set of mobile particles in the first interval. Particles that are mobile over both intervals were not considered for the analysis. We also generated a reference distribution $P_{\mathrm{m}}^{*}(r, \Delta \tau)$ of the minimum distance of each mobile particle in the second interval from a set of randomly chosen immobile particles in the first interval. From these two distributions, we computed $M(\Delta \tau)$, formally defined as

$$
M(\Delta \tau)=\frac{\int_{0}^{r_{\min }} P_{\mathrm{m}}(r, \Delta \tau) d r}{\int_{0}^{r_{\min }} P_{\mathrm{m}}^{*}(r, \Delta \tau) d r}
$$

where $r_{\min }$ corresponds to the first minimum of the radial pair correlation function $g(r)$.

Estimation of the trap potential. For $f_{\mathrm{p}} \geq 0.21$, where the mean separation between traps is of the order of $2 \sigma$, we ensured that the slowdown in dynamics was due to the presence of a trapped particle and not due to the potential of the trap. Towards this end, we computed the radial pair correlation function $g(r)$ of a dilute suspension $(\phi=0.26)$ of the smaller polystyrene particles with respect to a large reference polystyrene particle that was stuck to the glass coverslip. We then compared this $g(r)$ with the radial pair correlation of the same liquid in the presence of an optical trap centred on the stuck particle. We observed a slight enhancement in the first peak of $g(r)$ because of the presence of the trap (Supplementary Fig. 7), indicating that the trap contributes a small attractive potential in addition to freezing the particle on which it is centred. Using the dilute limit approximation to the potential of mean force ${ }^{42}$, we obtained a crude estimate of this excess potential and found it to be $\sim 0.15 k_{\mathrm{B}} T$. Further, beyond $r=1.1 \sigma$, the two correlation functions overlap, indicating that the potential of the trap decays well within the first nearest neighbour shell. This confirms that the glass transition seen in our experiments is purely an effect of the presence of pinned particles and is not influenced by the trap potential.

Dynamic susceptibility. To calculate the dynamic susceptibility $\chi_{4}\left(a_{\mathrm{p}}, t\right)^{43}$, we first computed the self-overlap function

$$
Q_{\mathrm{s}}\left(a_{\mathrm{p}}, t\right)=\frac{1}{N} \sum_{i=1}^{N} \exp \left(-\Delta r_{i}^{2}(t) / 2 a_{\mathrm{p}}^{2}\right)
$$

where $N$ is the total number of particles. The probe length scale $a_{\mathrm{p}}$ was varied from $0.03 \sigma$ to $\sigma$, where $\sigma$ corresponds to the first maximum of $g(r)$. We then extracted $\chi_{4}\left(a_{\mathrm{p}}, t\right)$ from the fluctuations in $Q_{\mathrm{s}}\left(a_{\mathrm{p}}, t\right)$

$$
\chi_{4}\left(a_{\mathrm{p}}, t\right)=N\left(\left\langle Q_{\mathrm{s}}\left(a_{\mathrm{p}}, t\right)^{2}\right\rangle-\left\langle Q_{\mathrm{s}}\left(a_{\mathrm{p}}, t\right)\right\rangle^{2}\right)
$$

The angular brackets indicate ensemble as well as time averaging. For the data shown in Supplementary Fig. 5, the value of $a_{\mathrm{p}}$ that maximized $\chi_{4}\left(a_{\mathrm{p}}, t\right)$ was chosen as the probe length scale.

\section{References}

1. Berthier, L. \& Biroli, G. Theoretical perspective on the glass transition and amorphous materials. Rev. Mod. Phys. 83, 587-645 (2011).

2. Biroli, G. \& Garrahan, J. P. Perspective: the glass transition. J. Chem. Phys. 138, 12A301 (2013)

3. Ediger, M. D. Spatially heterogeneous dynamics in supercooled liquids. Annu. Rev. Phys. Chem. 51, 99-128 (2000).

4. Hunter, G. L. \& Weeks, E. R. The physics of the colloidal glass transition. Rep. Prog. Phys. 75, 066501 (2012).

5. Adam, G. \& Gibbs, J. H. On the temperature dependence of cooperative relaxation properties in glass-forming liquids. J. Chem. Phys. 43, 139 (1965).

6. Hedges, L. O., Jack, R. L., Garrahan, J. P. \& Chandler, D. Dynamic order-disorder in atomistic models of structural glass formers. Science 323, 1309-1313 (2009). 
7. Cammarota, C. \& Biroli, G. Ideal glass transitions by random pinning. Proc. Natl Acad. Sci. USA 109, 8850-8855 (2012).

8. Franz, S. \& Parisi, G. Phase diagram of coupled glassy systems: a mean-field study. Phys. Rev. Lett. 79, 2486 (1997).

9. Kirkpatrick, T. R., Thirumalai, D. \& Wolynes, P. G. Scaling concepts for the dynamics of viscous liquids near an ideal glassy state. Phys. Rev. A. 40, 1045-1054 (1989)

10. Lubchenko, V. \& Wolynes, P. G. Theory of structural glasses and supercooled liquids. Annu. Rev. Phys. Chem. 58, 235-266 (2007).

11. Chandler, D. \& Garrahan, J. P. Dynamics on the way to forming glass: bubbles in space-time. Annu. Rev. Phys. Chem. 61, 191-217 (2010).

12. Ritort, F. \& Sollich, P. Glassy dynamics of kinetically constrained models. $A d v$. Phys 52, 219-342 (2003).

13. Jack, R. L. \& Berthier, L. Random pinning in glassy spin models with plaquette interactions. Phys. Rev. E. 85, 021120 (2012).

14. Keys, A. S., Hedges, L. O., Garrahan, J. P., Glotzer, S. C. \& Chandler, D. Excitations are localized and relaxation is hierarchical in glass-forming liquids. Phys. Rev. X., 1, 021013 (2011).

15. Elmatad, Y. S. \& Keys, A. S. Manifestations of dynamical facilitation in glassy materials. Phys. Rev. E. 85, 061502 (2012).

16. Candelier, R. et al. Spatiotemporal hierarchy of relaxation events, dynamical heterogeneities, and structural reorganization in a supercooled liquid. Phys. Rev. Lett. 105, 135702 (2010).

17. Vogel, M. \& Glotzer, S. C. Spatially heterogeneous dynamics and dynamic facilitation in a model of viscous silica. Phys. Rev. Lett. 92, 255901 (2004)

18. Bergroth, M. N. J., Vogel, M. \& Glotzer, S. C. Examination of dynamic facilitation in molecular dynamics simulations of glass-forming liquids. J. Phys. Chem. B 109, 6748-6753 (2005).

19. Candelier, R., Dauchot, O. \& Biroli, G. Building blocks of dynamical heterogeneities in dense granular media. Phys. Rev. Lett. 102, 088001 (2009).

20. Candelier, R., Dauchot, O. \& Biroli, G. Dynamical facilitation decreases when approaching the granular glass transition. Europhys. Lett. 92, 24003 (2010)

21. Bhattacharya, S. M., Bagchi, B. \& Wolynes, P. G. Facilitation, complexity growth, mode coupling, and activated dynamics in supercooled liquids. Proc. Natl Acad. Sci. USA 105, 16077-16082 (2008).

22. Weeks, E. R., Crocker, J. C., Levitt, A. C., Schofield, A. \& Weitz, D. A. Threedimensional direct imaging of structural relaxation near the colloidal glass transition. Science 287, 627-631 (2000).

23. Kegel, W. K. \& van Blaaderen, A. Direct observation of dynamical heterogeneities in colloidal hard-sphere suspensions. Science 287, 290-293 (2000).

24. Donati, C. et al. Stringlike cooperative motion in a supercooled liquid. Phys. Rev. Lett. 80, 2338 (1998).

25. Berthier, L. et al. Direct experimental evidence of a growing length scale accompanying the glass transition. Science 310, 1797-1800 (2005).

26. Kob, W., Roldán-Vargas, S. \& Berthier, L. Non-monotonic temperature evolution of dynamic correlations in glass-forming liquids. Nat. Phys 8, 164-167 (2012).

27. Marcus, A. H., Schofield, J. \& Rice, S. A. Experimental observations of nonGaussian behavior and stringlike cooperative dynamics in concentrated quasitwo-dimensional colloidal liquids. Phys. Rev. E 60, 5725 (1999).

28. Keys, A. S., Abate, A. R., Glotzer, S. C. \& Durian, D. J. Measurement of growing dynamical length scales and prediction of the jamming transition in a granular material. Nat. Phys. 3, 260-264 (2007).

29. Stevenson, J. D., Schmalian, J. \& Wolynes, P. G. The shapes of cooperatively rearranging regions in glass-forming liquids. Nat. Phys. 2, 268-274 (2006).

30. Starr, F. W., Douglas, J. F. \& Sastry, S. The relationship of dynamical heterogeneity to the Adam-Gibbs and random first-order transition theories of glass formation. J. Chem. Phys. 138, 12A541 (2013).

31. Gebremichael, Y., Vogel, M. \& Glotzer, S. C. Particle dynamics and the development of string-like motion in a simulated monoatomic supercooled liquid. J. Chem. Phys. 120, 4415 (2004).
32. Kob, W. \& Berthier, L. Probing a liquid to glass transition in equilibrium. Phys. Rev. Lett. 110, 245702 (2013).

33. Jack, R. L. \& Fullerton, C. J. Dynamical correlations in a glass former with randomly pinned particles. Phys. Rev. E 88, 042304 (2013).

34. Berthier, L. Overlap fluctuations in glass-forming liquids. Phys. Rev. E 88, 022313 (2013)

35. Biroli, G., Bouchaud, J.-P., Cavagna, A., Grigera, T. S. \& Verrocchio, P. Thermodynamic signature of growing amorphous order in glass-forming liquids. Nat. Phys. 4, 771-775 (2008).

36. Berthier, L. \& Kob, W. Static point-to-set correlations in glass-forming liquids Phys. Rev. E 85, 011102 (2012)

37. Charbonneau, P. \& Tarjus, G. Decorrelation of the static and dynamic length scales in hard-sphere glass formers. Phys. Rev. E 87, 042305 (2013).

38. Elmatad, Y. S., Jack, R. L., Chandler, D. \& Garrahan, J. P. Finite-temperature critical point of a glass transition. Proc. Natl Acad. Sci. USA 107, 12793-12798 (2010).

39. Speck, T. \& Chandler, D. Constrained dynamics of localized excitations causes a non-equilibrium phase transition in an atomistic model of glass formers. $J$. Chem. Phys. 136, 184509 (2012).

40. Nagamanasa, K. H., Gokhale, S., Ganapathy, R. \& Sood, A. K. Confined glassy dynamics at grain boundaries in colloidal crystals. Proc. Natl Acad. Sci. USA 108, 11323-11326 (2011).

41. Crocker, J. C. \& Grier, D. G. Methods of digital video microscopy for colloidal studies. J. Colloid. Interface. Sci. 179, 298-310 (1996).

42. Kepler, G. M. \& Fraden, S. Attractive potential between confined colloids at low ionic strength. Phys. Rev. Lett. 73, 356-359 (1994).

43. Dauchot, O., Marty, G. \& Biroli, G. Dynamical heterogeneity close to the jamming transition in a sheared granular material. Phys. Rev. Lett. 95, 265701 (2005).

\section{Acknowledgements}

We thank Gilles Tarjus, Sriram Ramaswamy, C. Patrick Royall, Francesco Sciortino and Chandan Dasgupta for useful discussions. We thank V. Santhosh for help in setting up the holographic optical tweezers, Mamta Jotkar for synthesizing silica colloids and Chandan Mishra and Amritha Rangarajan for synthesizing polystyrene colloids. S.G. thanks the Council for Scientific and Industrial Research (CSIR), India, for a Shyama Prasad Mukherjee Fellowship. K.H.N. thanks CSIR, India for a Senior Research Fellowship. R.G. thanks the International Centre for Materials Science (ICMS) and the Jawaharlal Nehru Centre for Advanced Scientific Research (JNCASR) for financial support and A.K.S. thanks Department of Science and Technology (DST), India, for support under J.C. Bose Fellowship.

\section{Author contributions}

S.G., K.H.N., R.G. and A.K.S designed the research. S.G. and K.H.N performed the experiments and analysed the data. S.G., K.H.N., R.G. and A.K.S wrote the paper.

\section{Additional Information}

Supplementary Information accompanies this paper at http://www.nature.com/ naturecommunications

Competing financial interests: The authors declare no competing financial interests

Reprints and permission information is available online at http://npg.nature.com/ reprintsandpermissions/

How to cite this article: Gokhale, S. et al. Growing dynamical facilitation on approaching the random pinning colloidal glass transition. Nat. Commun. 5:4685 doi: $10.1038 /$ ncomms5685 (2014). 